\title{
ONE-STEP AND TWO-STEP CALIBRATION OF A PORTABLE PANORAMIC IMAGE MAPPING SYSTEM
}

\author{
Ping-Chuan Wang ${ }^{\mathrm{a}}$, Po-Ching Tsai ${ }^{\mathrm{a}}$, Yung-Chuan Chen ${ }^{\mathrm{b}}$, Yi-Hsing Tseng ${ }^{\mathrm{a}, *}$
}

${ }^{\text {a } D e p t . ~ o f ~ G e o m a t i c s, ~ N a t i o n a l ~ C h e n g ~ K u n g ~ U n i v e r s i t y, ~ \# 1 ~ U n i v e r s i t y ~ R o a d, ~ T a i n a n, ~ T a i w a n ~-~}$

( peterson9623, fred1988715, cyc1130)@gmail.com, tseng@mail.ncku.edu.tw

${ }^{b}$ Dept. of Construction Science and Engineering, De-Lin Institute of Technology, \#1 Ln. 380, Qingyun Rd., Tucheng

Dist., New Taipei City, Taiwan - cyc1130@yahoo.com

\section{Commission I, WG I/3}

KEY WORDS: Photogrammetry, Close Range, Mapping System, Platforms, Calibration, Exterior Orientation.

\begin{abstract}
:
A Portable Panoramic Image Mapping System (PPIMS) is proposed for rapid acquisition of three-dimensional spatial information. By considering the convenience of use, cost, weight of equipment, precision, and power supply, the designed PPIMS is equipped with 6 circularly arranged cameras to capture panoramic images and a GPS receiver for positioning. The motivation for this design is to develop a hand-held Mobile Mapping System (MMS) for some difficult accessing areas by vehicle MMS, such as rugged terrains, forest areas, heavily damaged disaster areas, and crowed places etc. This PPIMS is in fact a GPS assisted close-range photogrammetric system. Compared with the traditional close-range photogrammetry, PPIMS can reduce the need of ground control points significantly. Under the condition of knowing the relative geometric relationships of the equipped sensors, the elements of exterior orientation of each captured image can be solved. However, the procedure of a system calibration should be done accurately to determine the relative geometric relationships of multi-cameras and the GPS antenna center, before the PPIMS can be applied for geo-referenced mapping. In this paper, both of one-step and two-step calibration procedures for PPIMS are performed to determine the lever-arm offsets and boresight angles among cameras and GPS. The performance of the one-step and two-step calibration is evaluated through the analysis of the experimental results. The comparison between these two calibration procedures was also conducted. The two-step calibration method outperforms the one-step calibration method in terms of calibration accuracy and operation convenience. We expect that the proposed two-step calibration procedure can also be applied to other platform-based MMSs.
\end{abstract}

\section{INTRODUCTION}

With the development of geomatics, spatial information accessing is becoming faster and more accurate and precise. Most of the current mobile mapping systems (MMSs) integrate multi-sensor systems for the acquisition of images, locations, orientation parameters, etc. The INS/GPS integrated systems are useful because of its rapid direct geo-referencing capability (Shin, 2005). Most MMSs use a vehicle as a platform (ElSheimy, 1996), which is convenient for the data collection of road reachable areas. However, many mapping areas are not allowed a vehicle to enter, such as rugged terrains, forest areas, heavily damaged disaster areas, crowed places etc. Ellum (2001) developed a Backpack Mobile Mapping System (BMMS), which integrates a camera, a GPS receiver, an inclinometer, and a compass. By considering the cost, weight of equipment, precision, and power supply, we propose an alternative version of BMMS, which is called a Portable Panoramic Image Mapping System (PPIMS). The PPIMS is a portable platform mounting six light cameras (for capturing panoramic images) and a GPS receiver. The GPS receiver can provide instant positioning for each picture taking position. Our prototype of PPIMS is less than $3 \mathrm{~kg}$, which can be hand-held to collect the spatial information for some areas that a vehicle-based MMS cannot enter. It is especially useful for emergency mapping of some disaster areas.
Before the PPIMS can be applied for geo-referenced mapping, the relative geometric relationships among the cameras and the GPS antenna should be calibrated. This procedure is commonly called system calibration, which is usually performed in an outdoor field to determine the lever-arm offsets and boresight angles (Ellum, 2001; Habib et al., 2011). The computation of system calibration is based on the bundle-adjustment theory, and the quality of calibration is highly dependent on the distribution of the control points in the calibration field. In order to obtain the exterior orientation of each image, every image should cover enough number of well distributed control points. It is really difficult to find a qualified outdoor calibration field. Although our outdoor calibration field contains more than 200 control points distributed on the walls of 4 surrounding buildings, it barely fulfils the calibration requirement. However, it is relatively easy to set up an indoor calibration field with a good distribution of control points. Therefore, instead of applying this one-step calibration method, this paper proposes a two-step calibration method. The first stage of the two-step method is to determine the relative geometric relationship of the cameras using an indoor panoramic calibration field. Then, the second step is to determine the lever-arm offset between the camera set and the GPS antenna center using a simple outdoor calibration field containing only a few control points.

\footnotetext{
* Corresponding author.
} 


\section{THE DESIGN OF PPIMS}

The PPIMS is in fact a GPS assisted close-range photogrammetric system, which is composed of three parts: the platform, the camera set and a GPS receiver. Fig. 1(a) shows a picture of the whole system and Fig. 1(b) shows that the system is set up on a surveying rod. The total weight of the platform, cameras and the GPS antenna is less than $3 \mathrm{~kg}$, so that it can be carried by a person for data collection.

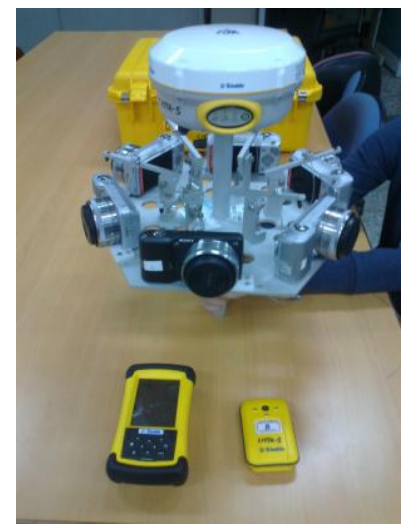

(a)

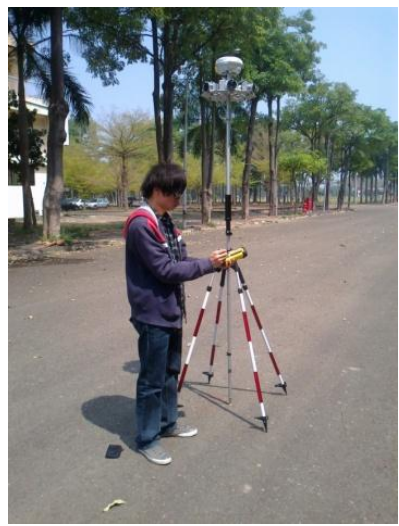

(b)
Figure 1. (a) A picture of the PPIMS; (b) The system is set up on a surveying rod.

\subsection{Platform}

The platform is designed to mount six cameras for simultaneously capturing six images to compose a panorama image. The platform, therefore, has a hexagon shape as shown in Fig. 2(a). The dimension of the platform is $40 \mathrm{~cm} \mathrm{x} 40 \mathrm{~cm} x$ $35 \mathrm{~cm}$. When the platform is installed on a surveying rod, the total height is about $2.4 \mathrm{~m}$. On the platform, there are six holes and locks to fix the six cameras. The pole in the middle of the platform has a standard screw to fix a GPS antenna. The six cameras can be triggered simultaneously with a mechanical device, which is composed of six string-controlled levers. The camera trigger mechanism can be operated with a string-pulling ring attached on the surveying rod as shown in Fig. 2(b).

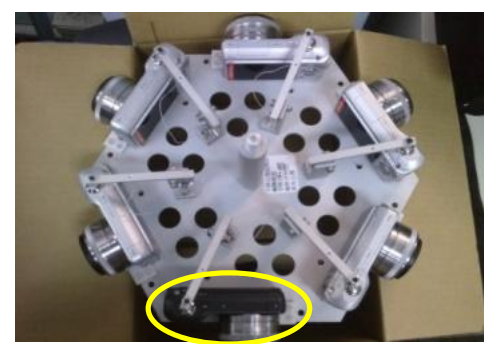

(a)

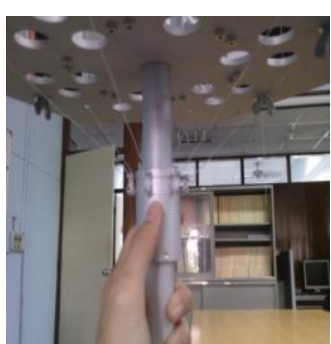

(b)
Figure 2. (a) The top view of the platform; (b) The bottom view of the platform.

\subsection{Cameras}

The PPIMS platform mounts six circularly arranged cameras to capture panoramic images. The adopted cameras should be light weight and should stable in taking high quality images. Among the commercial camera brands nowadays, we found that light-weight single-lens cameras are most qualified cameras. For our prototype of PPIMS, we chose the Sony Nex-3 camera (Fig. 3 ), which is a single-lens digital camera weights less than 300 grams. The camera uses a CMOS sensor. The sensor size is 23.4 $\mathrm{x} 15.6 \mathrm{~mm}$, and the image resolution is $4592 \times 3056$ pixels. The horizontal field of view (FOV) of the camera is about $65^{\circ}$ and the vertical FOV is about $43^{\circ}$. Each of the six cameras will always be installed in the same position on the platform. The six cameras are numbered from 0 to 5 . The camera numbered 0 is the black one indicated with a yellow circle in Fig. 2(a). The orientation of this camera will also be used as the orientation of the platform.

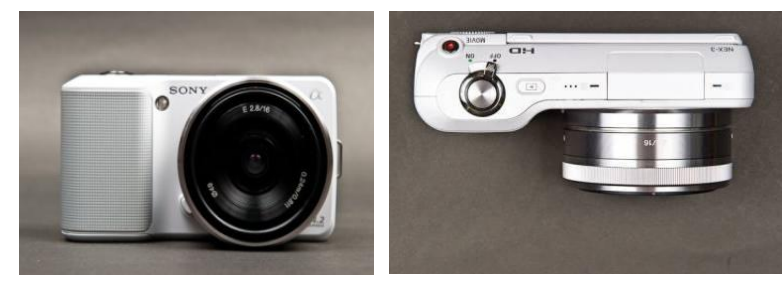

Figure 3. The adopted digital cameras (Sony Nex-3) for our prototype of PPIMS.

\subsection{GPS Receiver}

The PPIMS employs a GPS receiver to determine the position of picture taking in high accuracy. Fast positioning is also required for the use of PPIMS in an unstable environment. Under this circumstance, the GPS real-time kinematic (RTK) positioning method is adopted. Fortunately, the National Land Surveying and Mapping Centre (NLSMC) in Taiwan has set up a GPS network RTK system based on Virtual Reference Station (VRS) technology, which is called e-GPS (Electronic Global Positioning System). This system allows a user to perform RTK with a VRS GPS receiver through a network communication with a mobile phone to the e-GPS control center. This e-GPS system can determine a position instantly in the accuracy about $\pm 3 \sim 5 \mathrm{~cm}$. A Trimble VRS GPS receiver is adopted for our prototype of PPIMS as shown in Fig. 1(a). In case the e-GPS is not available, one can one nearby GPS base station to perform rapid relative positioning through a post processing of GPS baseline computation. It means that e-GPS is used for convenience but not for requirement.

If the GPS antenna and receiver are attached together, they should be mounted together on the platform and increase the weight of the hand-held unit. Some GPS receivers are connected with their control units and are separated from the antennas. By using this kind of GPS receivers, only the antenna needs to be mounted on the platform and the other parts can be carried with a bag or a backpack. Fig. 4 shows the use of this kind of GPS receiver.
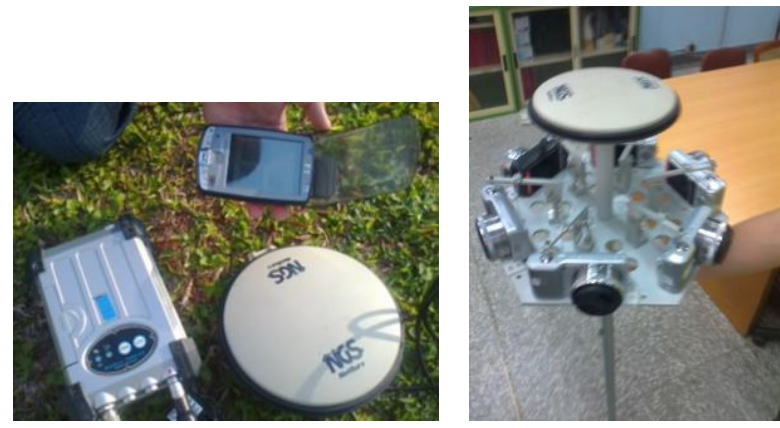

Figure 4. The use of antenna separated GPS receiver for the platform. 


\section{SYSTEM CALIBRATION}

Before the PPIMS can be applied for geo-referenced mapping, the calibration of camera interior orientation and a system calibration to determine the geometric relationship of the sensors must be performed. The calibration of camera interior orientation can be performed using regular close-range photogrammetric software. This paper mainly deals with the method of performing the system calibration.

The theory of system calibration involves the definition of applied coordinate systems. The PPIMS involved coordinate frames are the body frame, the camera frames and the mapping frame. If an MMS is integrated with GPS/INS, the body frame usually refers to the INS coordinate frame. Due to absence of the INS on PPIMS, the orientation of a referencing camera is adopted. The body frame of PPIMS is illustrated in Fig. 5. The body frame is defined by the phase center of GPS antenna as the origin, and the axes of the body frame is defined parallel to the photo coordinate axes of the referencing camera which is the black one numbered 0. The body frame of PPIMS is actually defined by GPS antenna position. It is, therefore, also called the GPS frame in this paper. The common definition of the camera frame applied in photogrammetric theory is adopted. Fig. 5 also shows the axes of the camera frames of PPIMS. The mapping frame is any local object coordinate system, which may be derived from some nearby control points.

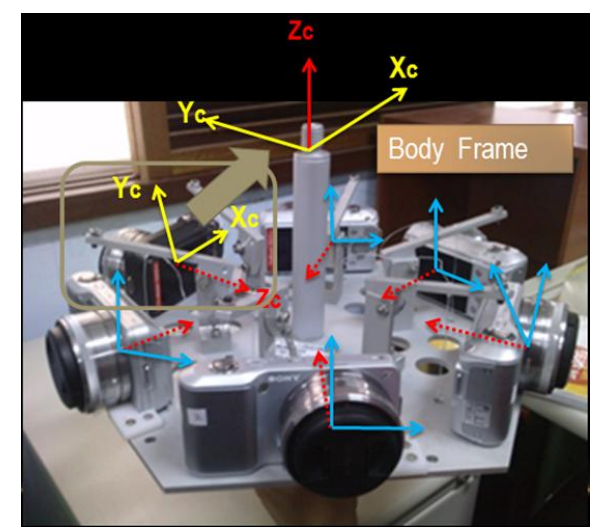

Figure 5. The body frame and the camera frames of PPIMS.

The relationship of the GPS antenna center, the camera perspective center, and an object point is shown in Fig. 6. The coordinates of an object point can be calculated through Equation 1 (Ellum, 2001). The rotation matrix $R_{c_{i}}^{M}$ of the $\mathrm{i}^{\text {th }}$ camera can be determined by Equation 2. The lever-arm offsets $r_{G P S}^{c_{i}}$ and boresight angles $R_{c_{i}}^{G P S}$ should be calibrated precisely.

$$
r_{P}^{M}=r_{G P S}^{M}-R_{c}^{M} r_{G P S}^{c}+\mu R_{c}^{M} r_{p}^{c}
$$

where $\quad r_{P}^{M}$ : object coordinates of point $\mathrm{P}$ in the mapping frame

$r_{G P S}^{M}$ :the position of the GPS in the mapping frame

$r_{G P S}^{c}$ :The position of the GPS in the camera frame

$r_{p}^{c} \quad$ :Camera coordinates of point $\mathrm{P}$ in the camera frame

$R_{c}^{M}$ :The rotation matrix from the camera frame

to the mapping frame

$$
R_{c_{i}}^{M}=R_{G P S}^{M} R_{c_{i}}^{G P S}
$$

where $\quad R_{c_{i}}^{G P S}$ :The rotation matrix from the $\mathrm{i}^{\text {th }}$ camera

frame to the GPS frame (body frame)

$R_{G P S}^{M}$ :The rotation matrix from the GPS frame

(body frame) to the mapping frame

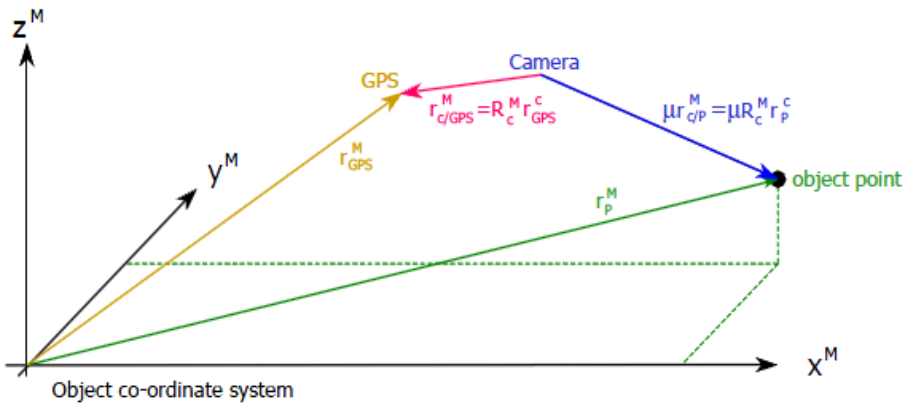

Figure 6. Relationship of GPS antenna center, camera perspective center, and an object point (Ellum, 2001).

For the experiments conducted in this study, an outdoor calibration field contains more than 200 control points distributed on the walls of 4 surrounding buildings was established for one-step calibration. An indoor calibration field with hundreds of photogrammetry targets was also set up for two-step calibration.

\subsection{One-step Calibration}

The one-step calibration is performed using the outdoor calibration field. The PPIMS took images on 4 stations in the calibration field. All control points on the images and manually selected conjugate image points were measured carefully. The computation of one-step calibration is based on the bundleadjustment theory. All exterior parameters including the perspective centers $\left(r_{c_{i}}^{M}\right)$ and orientation matrixes $\left(R_{c_{i}}^{M}\right)$ of the $\mathrm{i}^{\text {th }}$ camera be determined through a bundle-adjustment program. After the computation, the boresight angle $R_{c_{i}}^{G P S}$ can be calculated through Equation 3, which is derived from Equation 2. The GPS antenna position in the mapping frame, $r_{G P S}^{M}$, can be calculated using Equation 4 . The lever-arm, $r_{G P S}^{c_{i}}$, therefore can be calculated through Equation 5.

$$
\begin{aligned}
& R_{c_{i}}^{G P S}=\left(R_{G P S}^{M}\right)^{-1} R_{c_{i}}^{M} \\
& r_{c_{i}}^{M}=r_{G P S}^{M}-R_{c_{i}}^{M} r_{G P S}^{c_{i}} \\
& r_{G P S}^{c_{i}}=\left(R_{c_{i}}^{M}\right)^{-1}\left(r_{G P S}^{M}-r_{c_{i}}^{M}\right)
\end{aligned}
$$

Based on the bundle-adjustment theory, the quality of calibration is highly dependent on the number and distribution of tie and control points in the calibration field. In order to obtain the exterior orientation of each image, every image should cover enough numbers of well distributed control points. It is really difficult to find a qualified outdoor calibration field. Although our outdoor calibration field contains more than two hundred control points distributing on the walls of 4 
surrounding buildings, it barely fulfils the calibration requirement.

\subsection{Two-step Calibration}

It is relatively easy to set up an indoor calibration field with a good distribution of control points. Therefore, this paper proposes a two-step calibration method. The first stage of the two-step method is to determine the relative geometric relationship of the cameras using an indoor panoramic calibration field. Then, the second step is to determine the leverarm offset between the camera set and the GPS antenna center using a simple outdoor calibration field containing only a few control points.

In the first stage of two-step calibration, the PPIMS without the GPS receiver takes images on 4 stations in the indoor calibration field. Target codes are used in the indoor calibration field, which can be measured automatically by using a photogrammetric program (Fraser, 1997; Crock and Fraser, 2008). All exterior parameters including the perspective centers $\left(r_{c_{i}}^{M}\right)$ and orientation matrixes $\left(R_{c_{i}}^{M}\right)$ of the $\mathrm{i}^{\text {th }}$ camera can be determined through a bundle-adjustment program. After the computation, the boresight angle $R_{c_{i}}^{G P S}$ can be obtained from $R_{c_{i}}^{c_{0}}$ which is calculated through Equation 6, and $r_{c_{0}}^{c_{i}}$ can be calculated through Equation 8, which is derived from Equation 7.

$$
R_{c_{i}}^{c_{0}}=\left(R_{c_{0}}^{M}\right)^{-1} R_{c_{i}}^{M}
$$

where

$$
\begin{aligned}
& \text { the black one numbered } 0 \\
& r_{c_{i}}^{M}=r_{c_{0}}^{M}-R_{c_{i}}^{M} r_{c_{0}}^{c_{i}} \\
& r_{c_{0}}^{c_{i}}=\left(R_{c_{i}}^{M}\right)^{-1}\left(r_{c_{0}}^{M}-r_{c_{i}}^{M}\right)
\end{aligned}
$$$$
R_{c_{i}}^{c_{0}}: \text { The rotation matrix from the } \mathrm{i}^{\text {th }} \text { camera }
$$$$
\text { frame to the referencing camera which is }
$$

In order to calibrate the lever-arm offsets $r_{G P S}^{c_{i}}$, the second stage of the two-step calibration method should be performed as onestep calibration method using the outdoor calibration field. The boresight angle $R_{c_{i}}^{G P S}$ can be determined in the first stage of the two-step calibration method, it is not necessary to calculate the boresight angle $R_{c_{i}}^{G P S}$ in the second stage of the two-step calibration method. So compared with the one-step calibration method, it is can be expected that the precision of the boresight angle $R_{c_{i}}^{G P S}$ using the two-step calibration method will be improved.

\section{EXPERIMENTS}

\subsection{Test Fields}

Two test fields are designed to calibrate the lever-arm offsets and boresight angles among cameras and GPS: an indoor calibration field and an outdoor calibration field.
The indoor calibration field is shown in Fig. 7. Hundreds of photogrammetry targets are well distributed along the walls, ceiling, and floor. After capturing images at several stations, the interior orientation parameters (IOPs) and exterior orientation parameters (EOPs) can be calculated automatically by bundleadjustment software. The relative geometric relationships among cameras can be determined using the camera EOPs. During the first stage of the two-step calibration method, the lever-arm offsets and boresight angles among cameras can be determined precisely using the camera EOP results of indoor calibration.
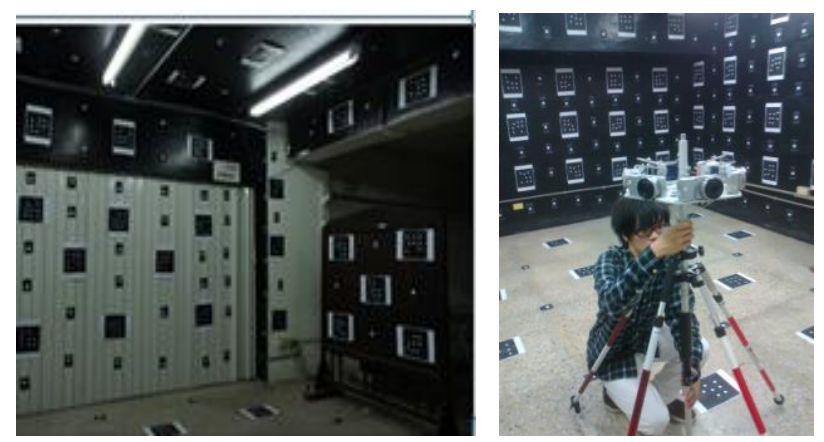

Figure 7. Indoor calibration field

The outdoor calibration field is shown in Fig. 8. There are 6 ground stations which coordinates were determined by using GPS. About 200 control points on the walls of four surrounding buildings were chosen. The coordinates of control points were determined by traditional surveying. After capturing images at several ground stations, hundreds of tie points were measured manually, and the EOPs of images could be calculated automatically by using bundle-adjustment software. Lever arm offsets among GPS antenna center and cameras can be determined using the coordinates of GPS stations and the EOPs of cameras. The one-step calibration method needs a lot of control points, but the two-step calibration just needs fewer control points because the relative geometric relationships of cameras has been calibrated precisely within the indoor calibration stage.
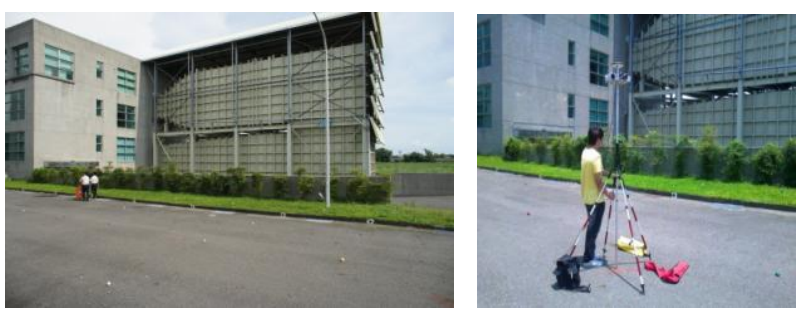

Figure 8. Outdoor calibration field

\subsection{Results and Analysis}

One-step calibration was performed in an outdoor calibration field, but two-step was performed both in an indoor calibration field and an outdoor calibration field. After one-step or two-step calibration, the lever-arm offsets and boresight angles among cameras can be determined. In order to compare one-step and two-step calibration results, relative distance from the perspective center of the referencing camera (camera " 0 ") to the other cameras were calculated (Fig. 9). An averaging process was performed to obtain mean values of relative distances, and their standard deviations were shown in table 1 . Compared with 
the one-step calibration method, the results of the two-step calibration procedure can largely improve the precision of the lever-arm offset among cameras.

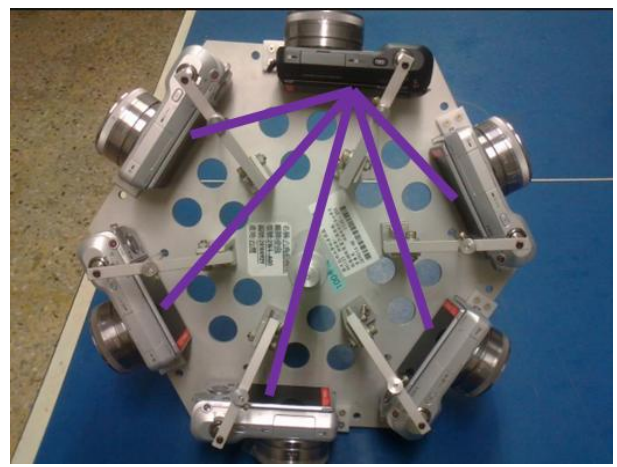

Figure 9. Relative distance from the perspective center of the reference camera (camera " 0 ") to the other cameras

Table 1. Standard deviations of relative distance from perspective center of referencing camera (camera " 0 ") to the other cameras (unit: $\mathrm{cm}$ )

\begin{tabular}{|c|c|c|c|c|c|}
\hline $\begin{array}{c}\text { Cameras } \\
\text { Method }\end{array}$ & $6-1$ & $6-2$ & $6-3$ & $6-4$ & $6-5$ \\
\hline One-step & 1.8 & 1.1 & 1.4 & 1.48 & 0.3 \\
\hline Two step & 0.1 & 0.1 & 0.1 & 0.1 & 0.1 \\
\hline
\end{tabular}

Error ranges of orientation angles of six cameras were shown in table 2. The error ranges of boresight angles were used to calculate the positioning precision in the 20 meters. Compared with one-step calibration method, the two-step calibration procedure can improve the precision of the boresight angles among cameras.

Table 2. Error ranges of boresight angles of six cameras and position precision in $20 \mathrm{~m}$

\begin{tabular}{|c|c|c|c|}
\hline Method & $\omega$ & $\varphi$ & $\kappa$ \\
\hline \multirow{2}{*}{ One-step } & $0.02 \sim 0.08^{\circ}$ & $0.04 \sim 0.09^{\circ}$ & $0.02 \sim 0.08^{\circ}$ \\
\cline { 2 - 4 } & $0.7 \sim 2.9 \mathrm{~cm}$ & $1.3 \sim 3.0 \mathrm{~cm}$ & $0.6 \sim 2.7 \mathrm{~cm}$ \\
\hline \multirow{2}{*}{ Two step } & $0.01 \sim 0.02^{\circ}$ & $0.01 \sim 0.02^{\circ}$ & $0.02 \sim 0.03^{\circ}$ \\
\cline { 2 - 4 } & $0.3 \sim 0.7 \mathrm{~cm}$ & $0.2 \sim 0.6 \mathrm{~cm}$ & $0.5 \sim 0.9 \mathrm{~cm}$ \\
\hline
\end{tabular}

After one-step or two-step calibration, the lever-arm offsets among the GPS antenna center and cameras can be determined. In order to compare one-step and two-step calibration results, relative distance from the GPS antenna center to the perspective centers of the cameras were calculated (Fig. 10). After averaging, standard deviations of relative distance from the GPS antenna center to the perspective centers of cameras were shown in table 3. Compared with the one-step calibration method, the two-step procedure didn't improve the precision of lever-arm offsets among the GPS antenna center and cameras.

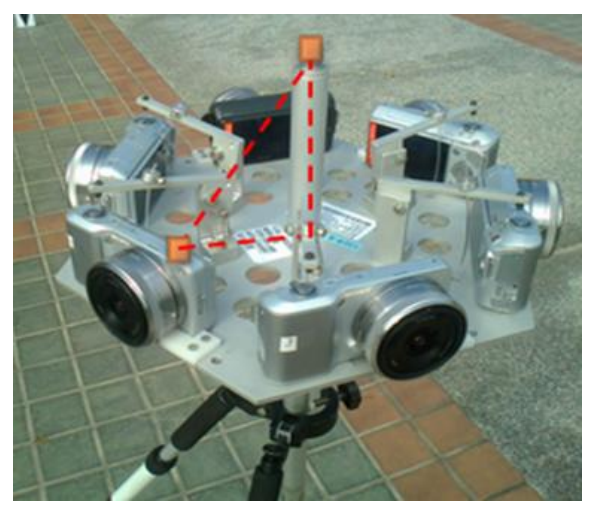

Figure 10. Relative distance from the GPS antenna center to perspective centers of cameras

Table 3. Standard deviations of Relative distance from GPS antenna center to perspective centers of cameras (unit: $\mathrm{cm}$ )

\begin{tabular}{|c|c|c|c|}
\hline Method & $\mathrm{dx}$ & $\mathrm{dy}$ & $\mathrm{dz}$ \\
\hline One-step & 0.9 & 2.0 & 1.5 \\
\hline Two step & 1.6 & 1.0 & 1.6 \\
\hline
\end{tabular}

The experimental results demonstrate that the two-step calibration procedure is superior to the one-step calibration procedure in terms of calibration accuracy among cameras. But the two-step calibration method still can't improve the precision of lever-arm offsets among GPS and cameras. For example, the two-step calibration procedure can improve the precision of lever-arm offset and boresight angle among the referencing camera (camera " 0 ") and camera " 1 ", but it still can't improve the precision of lever-arm offset among the GPS antenna and camera " 1 "

\section{CONCLUSIONS}

A PPIMS and its system calibration procedure are implemented and tested in this paper. Compared with a vehicle-based MMS, PPIMS provides the benefits of portability, simplified operation, overcoming the mapping limitations in relief terrain, and reduction of equipment cost. This system is expected to be conveniently applied to spatial information collection for disaster areas.

The system calibration of PPIMS can be done through the onestep or two-step calibration methods. The lever-arm offsets and boresight angles of PPIMS can be calculated after the calibration procedure. The two-step calibration method outperforms the one-step calibration method in terms of calibration accuracy and operation convenience. The experimental results show that the two-step calibration procedure largely improves the precision of boresight angles among the cameras although the precision of lever-arm offsets is not improved much. Also, the convenience and stability of the two-step calibration method are superior to the one-step calibration method. We expect that the proposed two-step calibration procedure can also be applied to other platformbased MMSs if their multi-cameras, GPS, and INS are rigidly mounted on a steady platform. 


\section{REFERENCES}

Cronk, S. and Frash, C. S., 2008. Hybrid Measurement Scenarios in Automated Close-Range Photogrammetry. The International Archives of the Photogrammetry, Remote Sensing and Spatial Information Science. Vol. XXXVII. Part B3b, pp.745-749, Beijing.

Ellum, C., 2001. The Development of a Backpack Mobile Mapping System. UCGE Reports No. 20159. Department of Geomatics Engineering, University of Calgary.

El-Sheimy, N., 1996. The Development of VISAT - A Mobile Survey System for GIS Applications. UCGE Reports No. 20101 Department of Geomatics Engineering, University of Calgary.

Fraser, C. S., 1997. Digital Camera Self-calibration, ISPRS Journal of Photogrammetry and Remote Sensing, Vol.52, pp. 149-159.

Habib, A., Kersting, A. P., Bang, K., Rau, J.,2011. A Novel Single-step Procedure for the Calibration of the Mounting Parameters of a multi-camera Terrestrial Mobile Mapping System. In Proceeding of the 7th International Symposium on Mobile Mapping Technology, Poland.

Shin, E. H., 2005. Estimation Techniques for Low-Cost Inertial Navigation. PhD Thesis, Department of Geomatics Engineering, University of Calgary, Calgary, Canada.

\section{ACKNOWLEDGMENT}

This research work was sponsored under the project grants: NSC 98-2211-E-224-MY2 and NSC 98-2211-E-219-MY2. The authors appreciate for the support of the National Science Council, Taiwan. 